\title{
Development of Real-Time Interworking between IEEE1888 and ECHONET Lite Standards for Building Energy Management System
}

\author{
Chayanon Sangumpai ${ }^{\mathrm{a}}$ and Chaodit Aswakul ${ }^{\mathrm{b} *}$ \\ Wireless Network \& Future Internet Research Unit, Department of Electrical Engineering, \\ Faculty of Engineering, Chulalongkorn University, Phayathai Road, Bangkok 10330, Thailand \\ E-mail: aChayanon.Sa@chula.ac.th, bChaodit.A@chula.ac.th (Corresponding author)
}

\begin{abstract}
This paper proposes the development of interworking proxy gateway for realtime data updates between IEEE1888 and ECHONET Lite standards in a building energy management system. Both IEEE1888 and ECHONET Lite are developed as open standards and hence a support for application and equipment development. IEEE1888 standard uses XML message format and is suitable for a wide range of machine-tomachine communications. ECHONET Lite standard is a highly accepted standard in Japan and with emerging supports by equipment manufacturers such as for air conditioners. This development of interworking proxy gateway in this research thus enhances the ability and flexibility in building energy management system to be compliant with different communication standards, which could be useful for both user and manufacturer sectors in the future.
\end{abstract}

Keywords: IEEE1888, ECHONET Lite, M2M communication, interworking, CU-BEMS.

ENGINEERING JOURNAL Volume 21 Issue 6

Received 29 August 2017

Accepted 16 October 2017

Published 31 October 2017

Online at http:/ / www.engj.org/

DOI:10.4186/ej.2017.21.6.1 


\section{Introduction}

Internet of Things (IoT) has recently been explored globally as popularized research topics as well as developed into new commercial products with the distinct capability that allows communication between various kinds of devices [1]. This concept involves connecting such things as sensors and actuators to the Internet-based infrastructure for the exchange of sensor reading data or actuation control signals. Due to expectedly huge number of devices that must be interconnected, an Io'T platform is potentially enabled by, not a single, but multiple machine-to-machine (M2M) communication technology or protocol standards [2]. Given the global crisis on world energy awareness, amongst possibilities of IoT application specifics, this paper is concerned with those viable IoT protocols within the scoped domain of energy management system in buildings or households.

In 2011, the Institute of Electrical and Electronics Engineers (IEEE) has initiated the so-called IEEE1888 standard for M2M communication technology for energy management systems with an inherently open communication architecture. In Japan, a main pilot project for this IEEE1888 proof-ofconcept system is the Ubiquitous Green Community Control Network (UGCCNet) [3]. The project aim is to establish communication and processing framework for overseeing energy consumption environments using sensors and actuators. UGCCNet users can control and monitor the devices remotely via the Internet with the TCP/IP protocol. The project has successfully integrated all necessary communicating devices and managed to raise international-level interests in the protocol adoption.

In Thailand, the IEEE1888 standard has been first introduced in the Chulalongkorn University's Building Energy Management System (CU-BEMS) project [4]. The project has been aimed at monitoring, controlling and analyzing actual energy consumption profiles as well as relevant building's ambient environments. Ongoing research based on the CU-BEMS demonstration site has ranged from the design and testing of smart metering technology [5], wireless ambient-sensor networking [6] and new Interactive Display as a Service (IDaaS) framework based on a smart in-house display [7] to the long-term evaluation of renewable energy source technologies [8]. The project also facilitates the data analytics research [9] to make people inside the buildings aware of comparative energy consumption levels at their own responsible areas. To ensure the interworking of all CU-BEMS components, all main devices have been developed to be compatible with the FETCH, WRITE and TRAP protocols of the IEEE1888 communication standard. Data are sent to gateways, a central data storage and web/mobile applications. Users of the building can access both raw data history as well as energy-profiling information from the project web portal [10].

In the emerging plethora of IoT standardization attempts, there exist however many other communication protocols in addition to the aforementioned IEEE1888 protocol. For instance, the work in [11] and [12] have aimed at studying the European IoT standard named ETSI M2M [13] and later known as OneM2M [14]. Particularly, their studies have tried to develop necessary interworking proxy gateways for the automatic data interchanging between the IEEE1888 and ETSI M2M standards in the CU-BEMS project. The data synchronization is carried out between the storage or database in the IEEE1888 domain and the repository in the ETSI M2M domain. In [11], the synchronization has been implemented periodically by using the WRITE protocol to send out data and the FETCH protocol to retrieve data with the IEEE1888 storage, and by using the CREATE method to record data and the RETRIEVE method to obtain data with the ETSI M2M repository. In a sequel work [12], the TRAP in place of FETCH protocols and the NOTIFY in place of RETRIEVE methods have been employed instead to achieve the real-time synchronous data linking without unnecessary latency introduced in the periodic data polling cycles.

Another widely accepted M2M communication protocol is the Japanese smart-house energy control standard based on the ECHONET Lite standard [15], [16]. This standard has been designed to simplify the establishment of M2M communication networks thanks to its target usage scenario inside households whose residents are unlikely to be technically literate enough for complex equipment setups. ECHONET Lite is compatible with electrical appliances of various brands. And, like the IEEE1888 goal, the ECHONET Lite standard has been developed as an open standard. However, to the best of our knowledge, despite of their potentials in being utilized within the same vicinity, these two standards are not known to be interoperable. A framework is still needed to ensure the interworking efficiency between the two protocols; and hence the main incentive of the research herein reported.

The objective of this paper is to look into the development of an interworking proxy gateway for realtime interworking between the IEEE1888 and ECHONET Lite standards. For completeness, in Sections 2 and 3 , the two communication protocol standards have first been revised with necessary terminologies needed later on in the design of their interworking mechanisms. Section 4 explains the proposed system 
architecture and details the actual proof-of-concept implementation for the newly developed interworking proxy gateway sitting at the boundary between the IEEE1888 and ECHONET Lite device domains. In Section 5, operational test results of the interworking proxy gateway have been highlighted within the departmental-scale CU-BEMS testbed facility. Section 6 finally concludes important lessons learnt in confirming the compatibility and individual protocol efficiency as quantifiable from the building energy management demonstration system of CU-BEMS.

\section{IEEE1888 Standard and Its Deployment in CU-BEMS Project}

The standard architecture of IEEE1888 [3] is composed of a registry and three main components (i.e. gateway, storage and application). Communication between these three components and the registry occurs via the TCP/IP network as illustrated in Fig. 1.

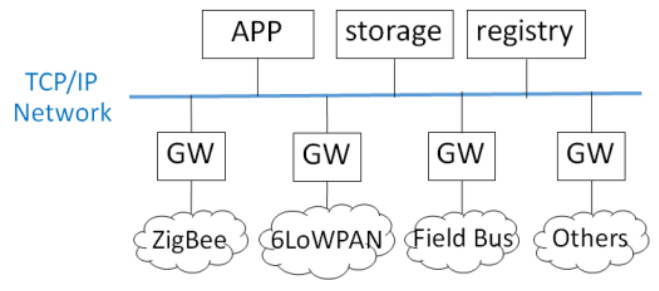

Fig. 1. IEEE1888 architecture [3].

Here, the application (APP) component is responsible for displaying necessary data from the sensors as well as allows users to enter control commands to activate installed actuators. The storage component keeps tracking of all the sensor readings and actuator commands as time-series data. Those data points in the storage can be read or written by other components with corresponding device identification given by the so-called point-id and point-set notions. The registry component helps keep records of all devices being instantiated and terminated at all time; therefore, the registry component plays a crucial controlling role of the operations of all other components within each IEEE1888 domain. One can view the registry as the operable entity of the control plane, and thus not the data plane operations. Lastly, the gateway component is intended for the accessibility controls of communicating devices between the basis IEEE1888 domain and any other domains talking in different communication protocol standards, including the ECHONET Lite in this paper.

In addition to standardizing the main components, the IEEE1888 standard defines the protocol primitives used for their communications. All communications are based on the Simple Object Access Protocol (SOAP) and three protocols have been given, namely, FETCH, WRITE and TRAP [3]. The FETCH protocol can be used for sending data queries and receiving data responses from one component to another component. The WRITE protocol can be used for writing time-stamped data values from one component to another component. And the TRAP protocol can be used for setting up data alert queries and sending out corresponding alerted data changes via a call-back mechanism. The conditions of data alerts are assigned in advance during the TRAP query phase.

Based on these IEEE1888 components and communication protocols, the CU-BEMS project [4] has developed a system that can monitor the ambient environmental parameters and measures the energy produced and used. The project aims at demonstrating the use of an M2M electricity management system in the building. The CU-BEMS system components are comprised of 200 temperature/humidity/light/human-motion sensors, 20 smart electricity meters, 10 air conditioning system actuators, together with a metering infrastructure for monitoring a standard electric-vehicle charging station, rooftop solar cell panels with the total generating capacity of $40 \mathrm{kWatts}$, and a small vertical-bladed wind turbine generator. The CU-BEMS APP components include an in-house developed web portal, an Android-based mobile application [10] as well as three IDaaS indoor monitors [7] each connected with a computing box and human-gesture detection capability with the Microsoft Kinect sensor to implement the interactive data visualization. 


\begin{tabular}{|c|c|c|c|c|c|c|}
\hline $\begin{array}{c}\text { CU-BEMS } \\
\text { Sensor }\end{array}$ & wireless & CU-BEMS & WRITE & IEEE1888 & FETCH & CU-BEMS \\
Gateway & XML & Storage & APP Display
\end{tabular}

Fig. 2. Example of wireless sensor communication in CU-BEMS.

Figure 2 illustrates a typical CU-BEMS example scenario whereby wireless sensors send their data first to be aggregated at an access gateway. In turn, the gateway converts the raw data bytes according to the standard IEEE1888 XML format before those data points are written to the central storage by using the WRITE protocol with repeated attempts if occasional writing failures may occur. The finally obtainable data stored can be read or displayed by queries from CU-BEMS APP components using the FETCH protocol. In this research, the TRAP protocol has been selected for data change alerts in developing the interworking proxy gateway, and the WRITE protocol has been used in sending data to the storage in order that the system can promptly communicate immediate changes in data values. This is useful in real-time system control situations such as IEEE1888-compatiable air conditioning system controller according to temperature and humidity data from sensors that can communicate rather by the ECHONET Lite standard. Communication using different standards is made possible by the interworking proxy gateway developed for this study. Additional details are given in Section 4.

\section{Revision of ECHONET Lite Standard}

ECHONET Lite [15] is a communication network standard aimed for sensors and actuators embedded in electrical appliances inside houses or buildings. The architecture of this standard is shown in Fig. 3. The boundary of a communication network administered as a unit with common properties is referred to as a domain. In each domain, a controller monitors and gives commands to all intra-domain devices. The controller also communicates between domains via a gateway working as an inter-domain node. The gateway is intended also to communicate with other systems outside the ECHONET Lite network, including the IEEE1888-compatible CU-BEMS in this research.

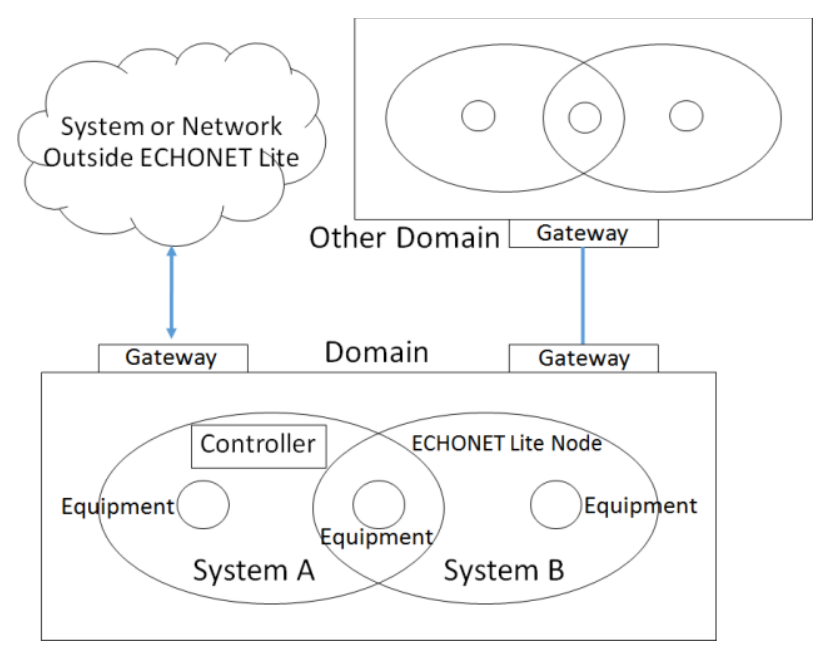

Fig. 3. ECHONET Lite architecture [15].

Followings are the main communication components defined by the ECHONET Lite standard. The ECHONET Lite node provides communications for all devices inside an ECHONET Lite network. An IP address is used to identify the node. The ECHONET Lite equipment or device can receive data from the ECHONET Lite node. It can also send data to the ECHONET Lite node. The ECHONET Lite gateway provides communications between an ECHONET Lite domain and other systems outside the ECHONET Lite network. It also provides communications between different domains each working in an ECHONET Lite network. 


\section{Proposed Interworking Proxy Gateway Design and Implementation}

The proposed system comprises of two domains: the domain in the CU-BEMS using the IEEE1888 standard and the other new domain using the ECHONET Lite standard. To make the two standards compatible, an interworking proxy gateway is needed to communicate data between the two standards. Figure 4 gives the overall architectural design of our test system. The interworking proxy gateway is comprised of two main abstract functional components. Firstly, an IEEE1888 gateway is responsible for interfacing with the IEEE1888 domain by exchanging necessary messages with the centralized storage of IEEE1888. Secondly, an ECHONET Lite gateway is responsible for interfacing with the ECHONET Lite domain. An ECHONET Lite domain typically consists of several ECHONET Lite nodes that communicate with each other within a local network. ECHONET Lite standard refers to each node as either self-node or other-node, where the self-node is the ECHONET Lite node under consideration from the viewpoint of an application software of interest, i.e. the interworking proxy gateway in this paper. In Fig. 4, the self-node abstraction has been explicitly included in referring to the communication functionality of the interworking proxy gateway with all other ECHONET Lite nodes.

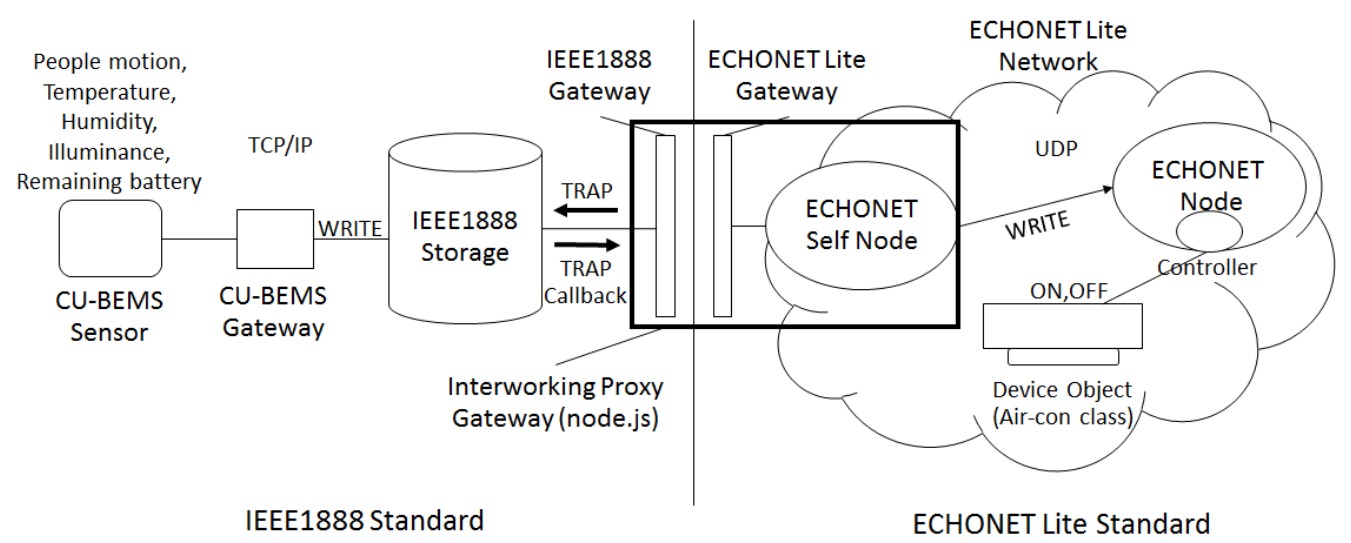

Fig. 4. Proposed interworking proxy gateway system architecture (IEEE1888 to ECHONET Lite domains).

The operations in Fig. 4 start from the domain of the IEEE1888 standard. Data detected by the sensor in the CU-BEMS system, such as motion, temperature, and humidity, useful for air conditioning control, are sent to the CU-BEMS gateway wirelessly. The data are converted into the standard XML format. Then the XML data are stored by using the WRITE protocol under the IEEE1888 standard based on the standard TCP/IP communication network. The interworking proxy gateway needs also send a TRAP alert query to the storage. When data changes, a TRAP callback can then be forwarded immediately to the domain of the ECHONET Lite to achieve the real-time data synchronization as desired. Finally, after the interworking proxy gateway receives the TRAP callback, the data is converted and can then be used subsequently. That is, a WRITE query under the ECHONET Lite standard is sent from self-node in the gateway to the node connected to electrical devices. In our testbed, the air conditioning system controller talking the ECHONET Lite standard can then be tuned of its operating point by relying on the ambient sensor readings from the IEEE1888 domain. All messages in ECHONET Lite standard in our implementation rely on the UDP communication framework.

In the current practice, an air conditioner must have been equipped from the manufacturer with an ambient sensor and the controller can communicate with the sensor without difficulties. Hence, it would not be necessary to have an additional interworking proxy gateway for communication implementation between the controller and sensor. However, recently, there emerges a new concept of smart air conditioning control (e.g. $[18,19])$, whereby the controller of air conditioner can be designed to take into account the reading of extra sensors from smart phones or wearable devices as well as additional ambient sensors installed to cover a large air-conditioned target area. The smartness invention is aimed to facilitate the novel modes of using the air conditioner to improve the personalized comforts of people and to enable the controllability precision in a critical area. Since extra sensors are not necessarily able to communicate in the same protocol as the air-conditioner controller, the interworking proxy gateway concept would be 
useful in those cases. Having an interworking proxy gateway is also a basis for future development towards the IoT framework.

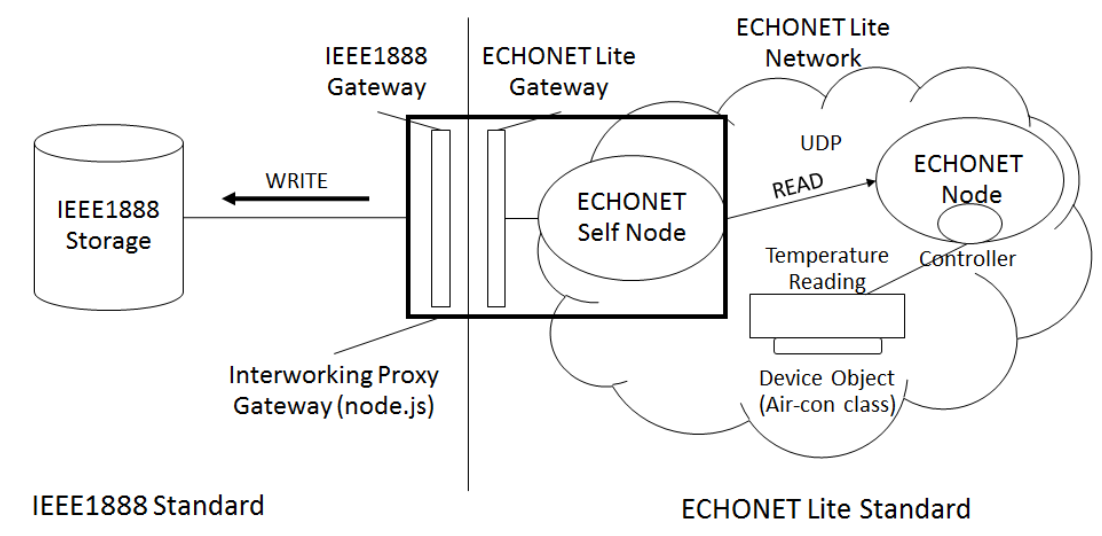

Fig. 5. Proposed interworking proxy gateway system architecture (ECHONET Lite to IEEE1888 domains).

Figure 5 illustrates the operations in the opposite direction to that of Fig. 4, i.e. the interworking being implemented to convert necessary data from ECHONET Lite domain to IEEE1888 domain. Conceptually, the operations start by the interworking proxy gateway sending a READ request to ECHONET Lite node to retrieve sensor data e.g. a temperature reading data from an air conditioning device. This request can be done periodically or upon the change of the sensing data of interest. After receiving the corresponding response carrying the new data, the interworking proxy gateway would accordingly change the received data format from ECHONET Lite to IEEE1888-based XML format. Finally, the prepared data can be further forwarded into the IEEE1888 domain with the interworking proxy gateway sending a WRITE message to update the data at the IEEE1888 storage.

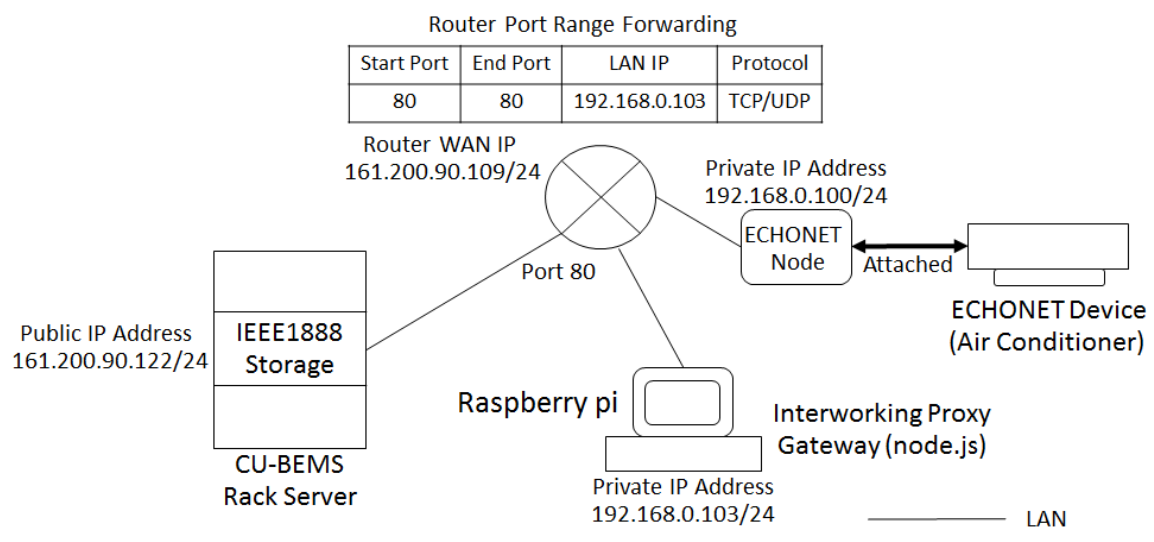

Fig. 6. The physical connections of the test system.

Physically, Fig. 6 depicts how all the components in both the IEEE1888 and ECHONET Lite domains as well as the interworking proxy gateway have been connected. The CU-BEMS IEEE1888-based storage, the interworking proxy gateway and the ECHONET Lite node have been connected to a router so that the three components can communicate directly with each other. The IP addresses of the components in the IEEE1888 standard domain have been set to public while those in the ECHONET Lite domain have been set to private. And the router is used to link between the two local area networks by a port forwarding scheme. 


\section{Test Results of Interworking Proxy Gateway}

The timing diagrams showing the interworking messages between the IEEE1888 standard and the ECHONET Lite standard via the interworking proxy gateway can be summarized in Figs. 7 and 8 . The operation sequence can be explained as follows.

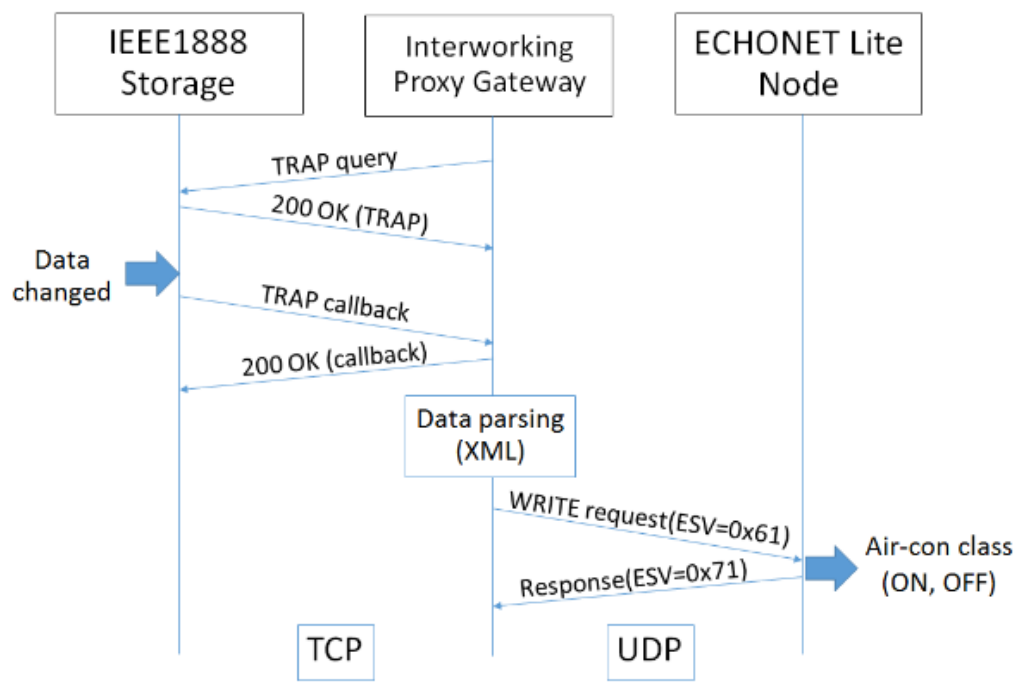

Fig. 7. Details of synchronization from IEEE1888 to ECHONET Lite.

In Fig. 7 , at first, the interworking proxy gateway sends a TRAP query for a data change alert to the IEEE1888 storage. Then, the IEEE1888 storage responds with 200 OK message. When there are changes in the data, the IEEE1888 storage responds with a TRAP callback message carrying the changed values. The interworking proxy gateway responds with 200 OK message after correctly receiving the TRAP callback message. After a protocol-conversion data parsing procedure, the data is reformatted and sent to the ECHONET Lite node e.g. for an air conditioning on/off control. The ECHONET Lite node responds to the interworking proxy gateway finally to confirm the accuracy of data received.

Likewise, Fig. 8 depicts the details of data-synchronization test from the ECHONET Lite to the IEEE1888 domains. Firstly, the interworking proxy gateway sends a READ query e.g. for a temperature reading from an ECHONET Lite node. The ECHONET Lite node responds then with a message to send the requested data reading to the interworking proxy gateway. After that, the interworking proxy gateway converts the received data into the IEEE1888-based XML format and sends a WRITE query using the WRITE protocol to the IEEE1888 storage. At last, the IEEE1888 storage responds with a message to the interworking proxy gateway after the data has been updated correctly.

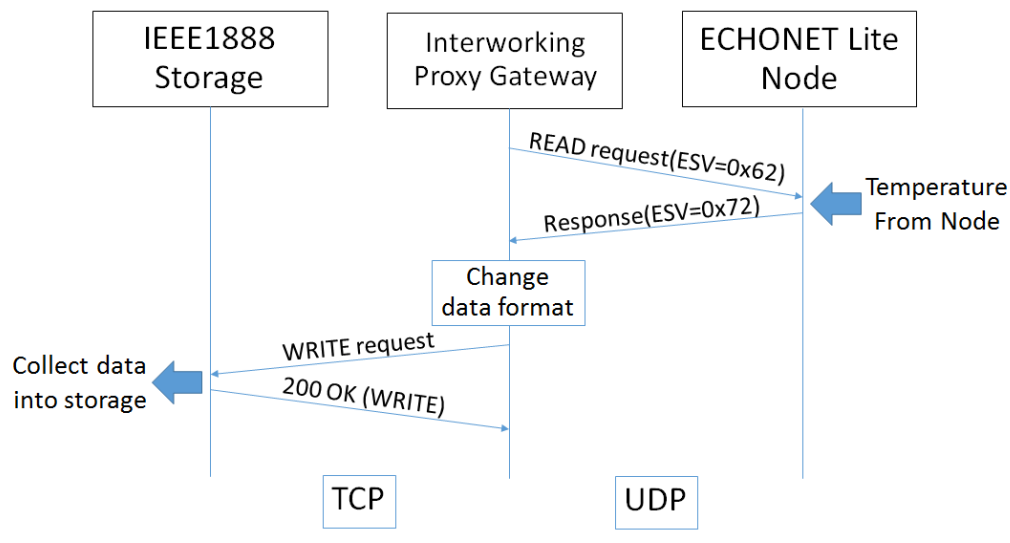

Fig. 8. Details of synchronization from ECHONET Lite to IEEE1888. 
The test system in both Figs. 7 and 8 have been developed on the node.js [17] platform. Here, a new test program using the SOAP package has been developed and the message formatting has been made compatible with the actual implementation of IEEE1888 standard in the existing CU-BEMS facility together with another developed program to communicate with a standard binary coding as specified in the ECHONET Lite standard.

Table 1. Size of messages used for communications from IEEE1888 to ECHONET Lite.

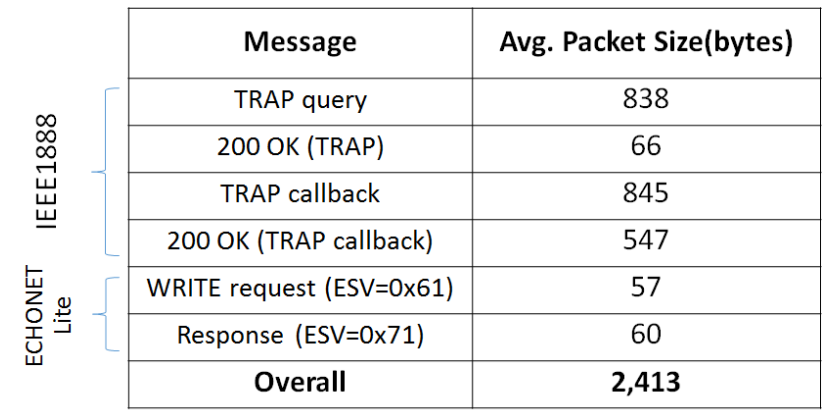

Table 2. Size of messages used for communications from ECHONET Lite to IEEE1888.

\begin{tabular}{|c|c|c|}
\hline \multirow{3}{*}{ 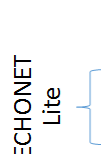 } & Message & Avg. Packet Size(bytes) \\
\hline & READ request $(E S V=0 \times 62)$ & 56 \\
\hline & Response $(\mathrm{ESV}=0 \times 72)$ & 60 \\
\hline $\begin{array}{l}w^{w} \\
\infty \\
\infty \\
\infty\end{array}$ & WRITE Protocol & 674 \\
\hline 出 & 200 OK (WRITE) & 66 \\
\hline & Overall & 856 \\
\hline
\end{tabular}

Tables 1 and 2 summarize the size of messages used for the communications between the three components. Since all the implementation is based on the actual operation of CU-BEMS, these provided information can be intended for a practical efficiency analysis of the two protocols. A test example has been conducted with an air conditioner's real sensor and actuator newly installed for the study using the ECHONET Lite standard. The sensor and actuator have been tested by using an existing sensor of the CU-BEMS project in our computer-cluster server room with the air conditioner. As seen in Tables 1 (and 2), to update per each data point, the messages of IEEE1888 standard are 2179 Bytes (624 Bytes, respectively) larger in size than those messages of ECHONET Lite standard. That means a higher bandwidth use by IEEE1888 than by ECHONET Lite. Expectedly, under the IEEE1888 protocol, the messages are sent in the XML format for human-level readability, whereas the ECHONET Lite uses binary coding intended for machine-level interpretability. For this reason, IEEE1888 standard can be more flexible in connecting with arbitrary devices which might exist in the system but have not yet been defined a priori in the ECHONET Lite binary coding standard. However, the ECHONET Lite standard can be more advantageous given that, in the future, electrical appliance manufacturers have decided to use the ECHONET Lite chips as their communicating module. As far as the data security is concerned, both the standards are at an equivalent level; the IEEE1888 can use the HTTPS and the ECHONET Lite can use an encrypted binary coding. 


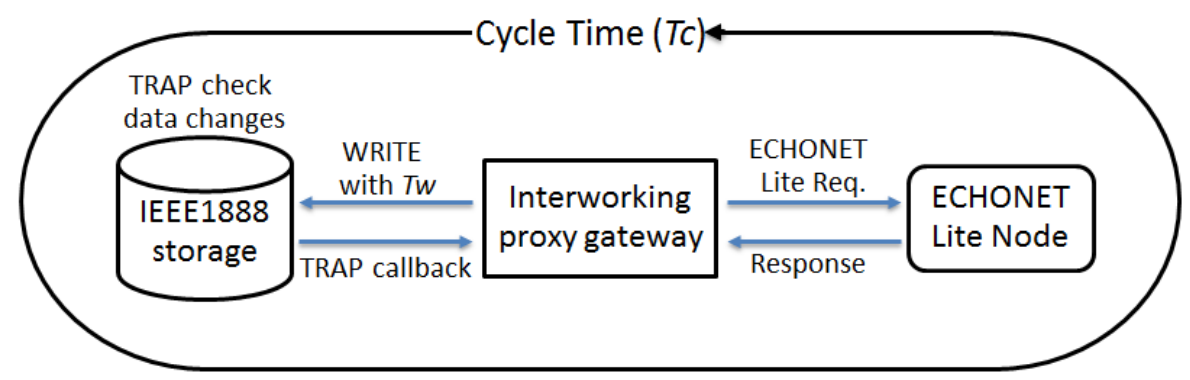

Fig. 8. Round-trip time delay or cycle time test scenario.

Data synchronization between the two standards has also been tested in terms of time delay performance. At the test beginning, the interworking proxy gateway sends a TRAP alert query to the storage to check for any data changes. Then WRITE queries, repeated at a varying time period of $T w$, by using the IEEE1888 WRITE protocol have been sent out to modify the trapped data in the storage. The modified data is then sent back with subsequent TRAP callbacks to the interworking proxy gateway. The converted data at the interworking proxy gateway can afterwards be sent to the ECHONET Lite node. After the ECHONET Lite node receives each query, the node responds with a message to the interworking proxy gateway. The time between when the interworking proxy gateway sends the WRITE queries and when the interworking proxy gateway later on receives the responses from the ECHONET node can be measured as herein called the round-trip time delay or cycle time $(T c)$ as shown in Fig. 8. This test has been conducted by sending the WRITE queries with different frequency per minute. The result is shown in Table 3.

Table 3. Effect of data synchronization frequency increase between IEEE1888 and ECHONET Lite.

\begin{tabular}{|c|c|}
\hline $\begin{array}{c}\text { WRITE } \\
\text { (times/minute) }\end{array}$ & $\begin{array}{c}\text { Average Cycle Time } \\
\text { Avg. Tc (millisecond) }\end{array}$ \\
\hline 1 & 178 \\
\hline 10 & 180 \\
\hline 20 & 219 \\
\hline 30 & 367 \\
\hline 50 & 424 \\
\hline
\end{tabular}

From Table 3, when increasing the frequency of the WRITE queries per minute from the interworking proxy gateway to the storage for data changes, the time for a full cycle of data synchronization between the two standards (Tc) has been found proportionally increased. The growing number of WRITE queries increases the traffic in the system, resulting in a potential load surge at the gateway processor. However, up to 50 times per minute, the resultant cycle time is still within a second, which should be acceptable for our CU-BEMS actuated control scenarios.

\section{Conclusion}

This study is concerned with a development and performance testing of interworking proxy gateway as a communication enabler between the IEEE1888 standard and the ECHONET Lite standard with the current focus upon the actual implementation integration within the CU-BEMS testbed. Confirmed by the test result based on the CU-BEMS scenarios, it has been found that the ECHONET Lite standard uses fewer bytes for the same set of messages when compared with the IEEE1888 standard. However, the IEEE1888 standard is more flexible in connecting with arbitrary devices which might exist in the system but have not yet been defined in the ECHONET Lite binary coding standard. The ECHONET Lite standard can be more advantageous in a smaller building or smart house domains, whose electrical appliance manufacturers use the ECHONET Lite chips as their communicating module. Regarding their 
expected applicability ranges, it is envisioned that the IEEE1888 standard with its flexibility can be suitable for a large network integration of small domains each communicating with ECHONET Lite or other available IoT standards. Development of interworking proxy gateways that can send messages between those relevant standards is therefore believed to be greatly beneficial in the scalable development of building energy management systems in the future.

\section{References}

[1] R. Khan, S. U. Khan, R. Zaheer, and S. Khan, "Future Internet: The Internet of things architecture, possible applications and key challenges," in 10th International Conference on Frontiers of Information Technology, 2012, pp. 257-260.

[2] M. Weyrich, J. P. Schmidt, and C. Ebert, "Machine-to-machine communication," IEEE Software, vol. 31, no. 4, pp. 19-23, July 2014.

[3] IEEE1888-2011, Standard for Ubiquitous Green Community Control Network, 2011.

[4] Energy Research Institute of Chulalongkorn University, "Smart grid technology for management of building electrical energy consumption," Technical Project Report, Energy Policy and Planning Office, Ministry of Energy, Thailand, September 2014.

[5] D. H. Le and W. Pora, "Development of smart meter for building energy management system based on the IEEE 1888 standard with Wi-Fi communication," in International Conference on Electronics, Information and Communications (ICEIC), 2014, pp. 1-2.

[6] T. Inthasut and C. Aswakul, "ZigBee wireless sensor network with IEEE1888 gateway for building energy management system," in International Conference Electronics Information and Communication (ICEIC), Kota Kinabalu, Malaysia, 2014, pp. 239-240.

[7] P. Khawsa-Ard and C. Aswakul, "IEEE1888 Interactive Display as a Service (IDaaS): Example in building energy management system," in IEEE 39th Annual Computer Software and Applications Conference, 2015, pp. 517-522.

[8] C. Ninagawa, H. Yoshida, S. Kondo, and H. Otake, "Data transmission of IEEE1888 communication for wide-area real-time smart grid applications," in International Renewable and Sustainable Energy Conference (IRSEC), 2013, pp. 509-514.

[9] K. Chooputtipong and C. Aswakul, "Development of data analytic program for building energy management system with wasted energy analysis using motion sensor," presented at 39th Electrical Engineering Conference (EECON-39), Phetchaburi, Thailand, 2016.

[10] CUBEMS: Chulalongkorn University Building Energy Management System. [Online]. Available: http://www.bems.ee.eng.chula.ac.th:9061/bems.web [Accessed: 14 February 2017]

[11] T. Klinpratum, C. Saivichit, A. Elmangoush, and T. Magedanz, "Toward interconnecting M2M/IoT standards: Interworking proxy for IEEE1888 standard at ETSI M2M platform," presented at 29th International Technical Conference on Circuit/Systems Computers and Communications (ITC-CSCC 2014), Phuket, Thailand, 2014.

[12] N. Kosolworrawattanakul, A. Elmangoush, T. Magedanz, and C. Aswakul, "Development of real-time data synchronization for IEEE1888 and ETSI M2M standards," IEICE Technical Report, Phuket, Thailand, 2014.

[13] ETSI M2M. [Online]. Available: http://www.etsi.org [Accessed: 14 February 2017]

[14] oneM2M. [Online]. Available: http://www.onem2m.org [Accessed: 14 February 2017]

[15] ECHONET Lite Specification, Version 1.11, Energy Conservation \& Homecare Network, 2014.

[16] T. Murakami, H. Sugimura, and M. Isshiki, "Application of ECHONET Lite which is open standard into energy management system," in IEEE International Conference on Consumer Electronics (ICCE), 2016, pp. $455-458$.

[17] S. Tilkov and S. Vinoski, "Node.js: Using JavaScript to build high-performance network programs," IEEE Internet Computing, vol. 14, no. 6, pp. 80-83, Nov. 2010.

[18] H. A. Özkan and A. Aybar, "A smart air conditioner in smart home," in 2016 IEEE 16th International Conference on Environment and Electrical Engineering (EEEIC), 2016, pp. 1-6.

[19] C. C. Cheng and D. Lee, "Smart sensors enable smart air conditioning control," Sensors, vol. 14, no. 6, pp. 11179-11203, 2014. 